\title{
Differential homeostatic regulation of glycinergic and GABAergic nanocolumns at mixed inhibitory synapses.
}

\section{Xiaojuan Yang ${ }^{1,2}$, Hervé Le Corronc ${ }^{3}$, Pascal Legendre ${ }^{3}$, Antoine Triller $^{1^{*}}$, Christian G Specht ${ }^{1,4^{*}}$}

1 Institute of Biology of the École Normale Supérieure (IBENS), CNRS, Inserm, PSL Research University, Paris, France

2 East China Normal University, Shanghai, China

3 Sorbonne University, Institute of Biology Paris Seine, Neuroscience Paris Seine, CNRS, Inserm, UPMC, Paris, France

4 Diseases and Hormones of the Nervous System (DHNS), Inserm, Le Kremlin-Bicêtre, France

*correspondence: antoine.triller@ens.psl.eu,christian.specht@inserm.fr

\section{Short title}

Differential regulation of GlyRs and $\mathrm{GABA}_{\mathrm{A}} \mathrm{Rs}$ at mixed synapses

\section{Key words}

GlyR, GABA $A_{A} R$, gephyrin, mixed inhibitory synapses, sub-synaptic domains (SSDs), trans-synaptic nanocolumns, super-resolution microscopy, direct stochastic optical reconstruction microscopy (dSTORM), two-color dSTORM.

\section{Highlights}

Alignment of sub-synaptic domains (SSDs) in trans-synaptic nanocolumns at inhibitory synapses Differential spatial organization of SSDs formed by GlyRs and $G_{A B A} R s$ at mixed inhibitory synapses Activity-dependent regulation of $\mathrm{GABA}_{\mathrm{A}}$ Rs but not GlyRs at mixed inhibitory synapses Gephyrin phosphorylation is compartmentalized in SSDs within the synaptic scaffold 


\section{Abstract}

Super-resolution imaging of synapses has revealed that key synaptic proteins are dynamically organized within sub-synaptic domains (SSDs). At mixed inhibitory synapses in spinal cord neurons, both GlyRs and $\mathrm{GABA}_{\mathrm{A}} \mathrm{Rs}$ reside at the same post-synaptic density (PSD). To examine how the different inhibitory receptors are organized and regulated, we carried out dual-color direct stochastic optical reconstruction microscopy (dSTORM). We found that endogenous GlyRs and $\mathrm{GABA}_{A} \mathrm{Rs}$ as well as their common scaffold protein gephyrin form SSDs that align with pre-synaptic RIM1/2, thus forming trans-synaptic nanocolumns. Strikingly, GlyRs and $G A B A_{A} R s$ occupy different sub-synaptic spaces, exhibiting only a partial overlap at mixed inhibitory synapses. When network activity was increased by pharmacological treatment using the $\mathrm{K}^{+}$channel blocker 4-aminopyridine (4-AP), the $G A B A_{A} R$ copy numbers of as well as the number of $G A B A_{A} R$ SSDs were reduced, while GlyRs remained largely unchanged. This differential regulation is likely the result of changes in gephyrin phosphorylation that preferentially occurred outside of the SSDs. The total gephyrin content was not altered by 4-AP application. The activity-dependent regulation of $G A B A_{A} R s$ versus GlyRs suggests that different signaling pathways control their respective sub-synaptic organization. Whereas gephyrin serves as a scaffold protein that upholds GlyR numbers at SSDs, it may act as a switch regulating $G A B A_{A} R s$ via its phosphorylation state. Taken together, our data reinforce the notion that the precise sub-synaptic organization of GlyRs, GABA $A_{A} R$ and gephyrin has functional consequences for the homeostatic regulation of mixed inhibitory synapses.

\section{Introduction}

The application of single molecule localization microscopy (SMLM) has provided new information about the nanoscale organization of synapses. Numerous post-synaptic proteins are heterogeneously distributed at synapses and compartmentalized into sub-synaptic domains (SSDs) (discussed in (Yang \& Specht, 2019)). At excitatory synapses, AMPARs assemble dynamically in SSDs that are stabilized by the scaffold protein PSD-95 (MacGillavry et al., 2013, Nair et al., 2013). At inhibitory synapses, gephyrin molecules also form SSDs, which are re-organized during inhibitory synaptic plasticity (Pennacchietti et al., 2017, Specht et al., 2013). Furthermore, Tang and colleagues have shown that post-synaptic SSDs at excitatory synapses are aligned with pre-synaptic SSDs, forming so-called trans-synaptic nanocolumns (Tang et al., 2016). This led to the proposal that subsynaptic domains are functional units underlying synaptic plasticity. 
Glycine receptors (GlyRs) and gamma-aminobutyric acid type A receptors ( $\left.G A B A_{A} R s\right)$ are the two main inhibitory receptors mediating fast inhibition in the central nervous system. In the spinal cord, GlyRs and $G_{A B A}$ Rs co-exist at the same post-synaptic density (PSD) during development and into adulthood (Chery \& de Koninck, 1999, Dumoulin et al., 2000, Legendre, 2001, Todd et al., 1996, Triller et al., 1987). These synapses are referred to as mixed inhibitory synapses. The corresponding neurotransmitters, glycine and GABA, are co-released from the same pre-synaptic vesicles (Jonas et al., 1998, Keller et al., 2001, O'Brien \& Berger, 2001). Glycinergic and GABAergic mIPSCs have distinct kinetics, the latter exhibiting slower decay times (Alvarez, 2017). Moreover, previous studies have shown that the lateral diffusion of synaptic GlyRs and $G A B A_{A} R s$ are differentially regulated at mixed inhibitory synapses by excitatory activity and microglial-dependent signaling (Cantaut-Belarif et al., 2017, Levi et al., 2008). These observations raise the possibility that the selective control of GlyRs and $G A B A_{A} R s$ could underlie the plasticity of mixed inhibitory synapses.

Gephyrin is the main scaffold protein at inhibitory synapses and a key player for both GlyR and $\mathrm{GABA}_{A} \mathrm{R}$ clustering. Biochemical studies have shown that gephyrin molecules form stable trimers through interactions of their G-domains, and that they can dimerize via their E-domains (Saiyed et al., 2007, Schrader et al., 2004, Sola et al., 2004). It has thus been proposed that gephyrin molecules form a hexagonal planar lattice below the post-synaptic membrane (Kneussel \& Betz, 2000). Gephyrin provides binding sites for both GlyRs and $G A B A_{A} R s$, even though the binding affinity for GlyRs is substantially higher than that of $\mathrm{GABA}_{A} \mathrm{Rs}$ (reviewed in (Tretter et al., 2012). The binding sites for the different receptor subunits in the gephyrin E-domain overlap, resulting in a competition between GlyRs and GABAARs for the same binding pocket of gephyrin at mixed inhibitory synapses \{Maric, $2011 \# 1815$ ). Furthermore, gephyrin molecules are prone to numerous post-translational modifications such as phosphorylation at multiple amino acid residues within the C-domain, which are important for the regulation of receptor binding and/or gephyrin clustering (Ghosh et al., 2016, Kalbouneh et al., 2014, Kuhse et al., 2012, Niwa et al., 2019, Tyagarajan et al., 2013, Tyagarajan et al., 2011).

In this study, we focused on the nanoscale organization of mixed inhibitory synapses in spinal cord neurons and investigated how endogenous GlyRs and $G_{A B} B_{A} R$ are regulated the sub-synaptic level using two-color dSTORM. Our data identify trans-synaptic nanocolumns comprising SSDs of RIM1/2, a component of the pre-synaptic active zone (AZ), as well as the post-synaptic scaffold protein gephyrin, GlyRs and $\mathrm{GABA}_{A} R s$. Interestingly, the two types of receptor were partially segregated, each dominating different sub-synaptic domains. While GlyR clustering was insensitive to short-term 
changes in network activity, $\mathrm{GABA}_{\mathrm{A}} \mathrm{Rs}$ were differentially regulated by neuronal activity, implying a key role in the homeostatic plasticity of mixed inhibitory synapses.

\section{Results}

\section{Trans-synaptic nanocolumns at inhibitory synapses in spinal cord neurons}

To probe the spatial relationship between pre- and post-synaptic elements at inhibitory synapses in spinal cord neurons, we conducted dual-color dSTORM imaging in native tissue. Semi-thin sections $(2 \mu \mathrm{m})$ of mouse spinal cord tissue were immuno-labeled with antibodies against the post-synaptic scaffold protein gephyrin and the pre-synaptic AZ protein RIM1/2, and imaged by dSTORM (Figure 1). The gephyrin clusters in dSTORM images exhibited a heterogeneous distribution, forming subsynaptic domains (SSDs) that are visible both in pointillist representations as well as rendered images (Figure $1 \mathrm{~A}-\mathrm{C}$ ). Two-color dSTORM images of gephyrin and RIM1/2 further revealed a close association between gephyrin SSDs and RIM1/2 SSDs (Figure 1D). This alignment of pre- and postsynaptic SSDs is reminiscent of trans-synaptic nanocolumns that have been identified at excitatory synapses in primary cultured neurons (Tang et al., 2016). The identification of gephyrin SSDs and their alignment with pre-synaptic release sites in vivo points to a possible role for synaptic function.

For a closer characterization of the spatial organization we made use of cultured spinal cord neurons for our subsequent experiments. Two-color dSTORM imaging of synapses in cultured neurons was conducted on the target proteins in a pair-wise manner (see Methods). To quantify the pre- and post-synaptic organization along the trans-synaptic axis, super-resolution images of synapses with side view profiles were selected from the rendered dSTORM images. These images revealed a heterogeneous distribution of RIM1/2, gephyrin (antibody mAb7a), GlyRs and $G A B A_{A} R s$, all of which formed distinctive SSDs at inhibitory synapses (Figure 2). The number of SSDs per synaptic cluster for these proteins was generally two to four, and the SSD counts increased with the synaptic cluster

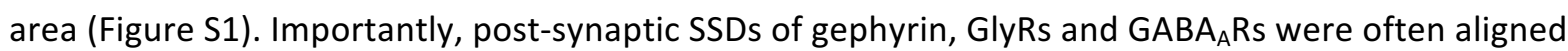
with pre-synaptic RIM1/2 SSDs (Figure 2).

We measured the distances between the paired pre-synaptic SSDs and post-synaptic SSDs (Figure 2A1-C1, see Methods). The average distance between SSDs of pre-synaptic RIM1/2 and postsynaptic gephyrin and was $99 \pm 3 \mathrm{~nm}$ (mean \pm SEM, from 48 synapses). The distance between SSDs of RIM1/2 and $\mathrm{GABA}_{A}$ Rs was very similar, measuring $102 \pm 5 \mathrm{~nm}$ (from 16 synapses), however, the distance between RIM1/2 and GlyR SSDs was only $74 \pm 4 \mathrm{~nm}$ (from 30 synapses). This difference is likely due to the fact that GlyRs were labeled using antibodies against an extracellular epitope, 
whereas $G A B A_{A} R s$ were labeled at an intracellular epitope. The apparent distance of $28 \mathrm{~nm}$ between the extracellular and the intracellular epitope is consistent with the longitudinal dimension of receptor complexes ( $20 \mathrm{~nm}$; (Patriarchi et al., 2018)) and the size of a single antibody (12-15 nm; (Maidorn et al., 2016)). These measurements also illustrate that the sequential immuno-labeling with classical antibodies does not add cumulative distances to the distance measurement, probably due to the random orientation of the antibodies.

Next, we quantified the degree of alignment between pre- and post-synaptic structures, expressed as the ratio of paired SSDs over the total SSDs for each target protein (Figure 2A2-C2). The majority of gephyrin SSDs were aligned with RIM1/2 SSDs (0.91 \pm 0.03 , mean \pm SEM), and most RIM1/2 SSDs were in turn also aligned with SSDs of gephyrin $(0.73 \pm 0.04)$. Similarly, RIM1/2 SSDs were aligned with GlyR and $G A B A_{A} R$ SSDs $(0.87 \pm 0.03$ and $0.78 \pm 0.06$, respectively) and vice versa $(0.79 \pm 0.04$ and $0.78 \pm 0.07$, respectively). These results confirm the existence of trans-synaptic nanocolumns at inhibitory synapses in cultured spinal cord neurons.

Trans-synaptic nanocolumns consisting of RIM1/2 and gephyrin SSDs may recruit both GlyRs and $\mathrm{GABA}_{A} \mathrm{Rs}$ at mixed inhibitory synapses. This is consistent with a recent study using STED and SIM super-resolution imaging, showing that gephyrin SSDs as well as $G A B A_{A} R$ SSDs were closely associated with RIM1/2 SSDs at hippocampal and cortical synapses (Crosby et al., 2019). Given that trans-synaptic nanocolumns were also observed at excitatory synapses (Haas et al., 2018, MacGillavry et al., 2013, Tang et al., 2016), it appears likely that they have a common functional role in the fine-tuning of synaptic transmission.

\section{Partial overlap of GlyR and GABA $A_{A}$ SSDs at mixed inhibitory synapses}

It was shown that most inhibitory synaptic boutons in cultured spinal cord neurons are apposed to PSDs containing both GlyRs and $G_{A B A_{A} R s}$ (Dumoulin et al., 2000). Using two-color dSTORM, we therefore compared how GlyRs and $G_{A B A_{A} R s}$ were organized at these mixed inhibitory synapses.

In multi-color dSTORM experiments, the choice of fluorescent dyes is one of the main factors determining the quality of the reconstructed super-resolution images (Dempsey et al., 2011, van de Linde et al., 2011). In addition, it is essential to achieve dense labeling, which is very challenging in the case of synaptic proteins, particularly neurotransmitter receptors (discussed in (Yang \& Specht, 2020)). We tested different antibody and dye combinations for the detection of GlyR and $G A B A_{A} R$ clusters in two-color dSTORM. The best combination was to label $G_{A B A} A_{A}$ Rs with secondary antibodies conjugated with Alexa Fluor 647 (A647) and GlyRs with Cy3B (Figure S2), since the number of detections in dSTORM was closely correlated with the cluster intensity in the 
epifluorescence images (Figure S2A5-B5). The detection efficiency of this dye combination was also relatively good when the amount of receptor labeling was low (Figure S2C1-D5).

Using this labeling protocol, we compared the spatial organization of GlyRs and $G A B A_{A} R s$ at mixed inhibitory synapses in cultured spinal cord neurons. Receptor clusters in rendered dSTORM images were highly variable, even though the shape and size of the overall synaptic structure was similar (Figure 3A-B upper panels). GlyRs and $\mathrm{GABA}_{A}$ Rs were not homogeneously distributed at the PSDs and displayed limited co-localization. To evaluate the level of overlap between the receptor domains, we calculated the overlapped fraction that is the area containing both GlyRs and $G A B A_{A} R s$, divided by the total cluster area (Figure $3 \mathrm{C}$ ). The overlapped fraction was generally well below $50 \%$. Furthermore, we segmented synaptic clusters into sub-synaptic domains. GlyR SSDs and GABA ${ }_{A} R$ SSDs did not always overlap in the binary images (Figure 3A-B lower panels). As judged by the overlap fraction, more than half of the synapses (55\%) did not show any co-localization between GlyR SSDs and GABA ${ }_{A} R$ SSDs (Figure 3D). In the remainder of the synapses the SSDs of GlyRs and $G_{A B A_{A}} R s$ overlapped partially, but were often dominated by one or the other of the two receptors. As a result, the average overlapped fraction of SSD area was only about $9 \%$ at these synapses.

The low level of overlap between GlyR SSDS and GABA ${ }_{A} R$ SSDs was unexpected, since both receptors were well aligned with RIM1/2 SSDs ( $0.79 \pm 0.04$ and $0.78 \pm 0.07$, respectively). Consequently, the overlap between GlyR and GABA $A_{A} R$ SSDs should have been around $62 \%(0.79 * 0.78 * 100 \%)$. This apparent discrepancy can be attributed to several factors. Firstly, the synapses in this experiment were not chosen based on their orientation. Instead, this analysis was performed on hundreds of synapses, regardless of whether they were seen in side view or en face. Second, the labeling of GlyRs at an extracellular epitope and of $\mathrm{GABA}_{\mathrm{A}} \mathrm{Rs}$ at an intracellular epitope may increase the distance between the fluorophore detections in the two channels, thus lowering the apparent overlap. Nevertheless, it appears that GlyRs and $\mathrm{GABA}_{A}$ Rs form distinct SSDs that are often separated or dominated by one of the two receptor types.

\section{Differential regulation of GlyRs and $\mathrm{GABA}_{\mathrm{A}} \mathrm{Rs}$ in response to altered network activity}

Since GlyRs and $\mathrm{GABA}_{A}$ Rs occupy different sub-synaptic compartments, we hypothesized that they may be controlled through different clustering mechanisms. To investigate whether GlyRs and $\mathrm{GABA}_{\mathrm{A}} \mathrm{Rs}$ were differentially regulated by neuronal activity, we adopted a pharmacological approach. Spinal cord neurons were treated either with tetrodotoxin (TTX) or with 4-aminopyridine (4-AP) to reduce or increase the neuronal network activity, respectively. We observed few calcium signals in neurons under control conditions, suggesting a low basal activity in cultured spinal cord neurons. 
TTX completely abolished the calcium signals, while 4-AP increased the firing frequency but did not change the amplitude of the calcium transients (Figure S3).

To examine whether GlyRs and $\mathrm{GABA}_{\mathrm{A}} \mathrm{Rs}$ were affected by changes in network activity, neurons were treated with TTX and 4-AP for one hour, then fixed and triple labelled with antibodies against GlyRs, $\mathrm{GABA}_{\mathrm{A}} \mathrm{Rs}$ and gephyrin. Epifluorescence images in the three channels were taken with conventional fluorescence microscopy (Figure 4A). Synaptic clusters were detected by generating masks based on the gephyrin labeling. The size of the gephyrin puncta (area in pixels) did not differ between the TTX and 4-AP conditions (Figure 4B). The gephyrin masks were then used to measure the intensities of the different associated synaptic receptors. Interestingly, $G A B A_{A} R$ but not GlyR intensity was significantly decreased after 4-AP treatment, compared to the TTX condition that served as control condition in these experiments (Figure 4C, Figure S4). In order to directly compare how the two receptors reacted at the same PSDs, we calculated the ratio of $G A B A_{A} R / G l y R$ intensity for each synapse. Lower $\mathrm{GABA}_{A} \mathrm{R} / \mathrm{Gly} \mathrm{R}$ ratios under 4-AP confirmed that higher network activity decreases synaptic $G A B A_{A} R$ levels but had no or little effect on GlyRs at the same PSDs (Figure 4D). To further investigate these changes in relation to the sub-synaptic organization of GlyRs and $G A B A_{A} R s$, we then conducted super-resolution imaging in neurons exposed to altered network activity.

\section{Activity-dependent changes of the sub-synaptic organization of GlyRs and $G_{A B A_{A}} R s$}

Two-color dSTORM imaging of GlyRs and GABA ${ }_{A} R s$ was performed after one hour of TTX or 4-AP bath application. In order to compare the number of detections of GlyRs and $G A B A_{A} R s$ within the same synapse, the rendered dSTORM images of GlyR and GABA $A_{A} R$ clusters were combined to produce total synaptic clusters that we refer to as integrated receptor clusters (Figure 5A). These clusters were binarized to produce a mask of the overall synapse, within which the number GlyR and $G A B A_{A} R$ detections were determined (Figure $5 B$ ). After 4-AP treatment, the number of $G A B A_{A} R$ detections was significantly decreased, while that of GlyRs did not change (Figure 5C-D). In agreement with the data shown in Figure 4, the ratios of $G A B A_{A} R / G$ lyR detections per synapse were reduced, confirming that this phenomenon occurred within individual PSDs (Figure 5E). We also saw a reduction in the area of the integrated receptor clusters following 4-AP treatment (Figure S5A). Together, these results established that the copy numbers of $G A B A_{A} R s$ and GlyRs at synapses are differentially regulated by network activity.

Next, we segmented the integrated receptor clusters into SSDs, referred to as receptor SSDs, and counted the number of detections of GlyRs and $G_{A B A}$ Rs within each of them (Figure 5F-G). This allowed us to compare the relative changes of GlyRs and $G_{A B A} R s$ within the same sub-synaptic 
domains. The size of the receptor SSDs was not affected by the treatments (Figure S5B). However, the number of $G A B A_{A} R$ detections was decreased by $4-A P$, whereas the number of $G l y R$ detections did not change (Figure $5 \mathrm{H}-\mathrm{I}$ ). The reduction of $\mathrm{GABA}_{A} \mathrm{R}$ relative to GlyR copy numbers occurred within the same SSDs, as shown by the ratio of detections per SSD (Figure 5J). These results show that the activity-dependent re-distribution of $\mathrm{GABA}_{\mathrm{A}} \mathrm{Rs}$ versus GlyRs occurs also at the sub-synaptic level.

The spatial organization at mixed inhibitory synapses was further assessed by quantifying the number of GlyRs and GABA ${ }_{A} R s$ SSDs separately (Figure 6). The number of integrated receptor SSDs per synapse was reduced after 4-AP treatment (Figure 6A), but not their size (Figure S5B). This reduction could be attributed to a large extent to $G A B A_{A} R s$ rather than GlyRs, since the number of $\mathrm{GABA}_{A} \mathrm{R}$ SSDs was strongly reduced (49\% of the TTX condition, $p<0.0001$, Mann-Whitney test), compared to that of GlyR SSDs ( $88 \%$ of TTX condition, $p<0.01$ ) (Figure $6 B-C$ ). This further indicates that the spatial organization of inhibitory receptor domains of GABARs and GlyRs is differentially regulated at mixed inhibitory synapses.

\section{Sub-synaptic regulation of gephyrin phosphorylation}

In order to explore a possible mechanism by which synaptic activity could differentially regulate GlyRs and $G A B A_{A} R s$ at mixed inhibitory synapses, we studied the phosphorylation of gephyrin at the regulatory site S270 (reviewed in (Alvarez, 2017, Specht, 2020)). We took advantage of the fact that the monoclonal antibody mAb7a specifically recognizes phosphorylated gephyrin (Kuhse et al., 2012). Hence we examined how this post-translational modification responded to neuronal activity changes.

We first compared the amount of total gephyrin (probed by the polyclonal antibody rbGPHN) and of S270 phosphorylated gephyrin (probed by mAb7a) using conventional fluorescence microscopy

(Figure 7A-C). The colocalization of rbGPHN and mAb7a puncta did not differ between the TTX and 4AP conditions (Figure S6A). Using a binary mask of rbGPHN clusters, we measured the fluorescence intensity of both rbGPHN and mAb7a signals at the same puncta. The fluorescence intensity of mAb7a but not rbGPHN was significantly decreased by 4-AP treatment (Figure 7B, also see Figure S4A). This was also true at individual synapses, as indicated by the fluorescence intensity ratio (Figure 7C). The unchanged total number of gephyrin molecules at synapses suggests that the gephyrin scaffold persists during changes in network activity, while the $\$ 270$ phosphorylation is dynamically regulated. We therefore explored with dSTORM the sub-synaptic distribution of S270 phosphorylated gephyrin after 4-AP treatment. The number of mAb7a detections per synapse was 
decreased by 4-AP (Figure 7D), as expected from the reduction of mAb7a labeling seen with conventional fluorescence microscopy (Figure 7B). At the sub-synaptic level, neither the number of detections of pS270 gephyrin (mAb7a) per SSD nor the number of SSDs were altered following 4-AP treatment (Figure 7E-F). Moreover, the size of pS270 gephyrin SSDs did not change (Figure S6B). It thus appears that pS270 gephyrin is concentrated within regions of the synaptic scaffold that are not affected by 4-AP treatment, suggesting that S270 de-phosphorylation occurs predominantly at gephyrin molecules located outside of these SSDs.

\section{Discussion}

The heterogeneity of synaptic protein distribution has been observed in many instances using superresolution imaging with different techniques such as SMLM, STED and SIM (Crosby et al., 2019, MacGillavry et al., 2013, Nair et al., 2013, Pennacchietti et al., 2017, Specht et al., 2013, Wegner et al., 2018). The sub-synaptic domain (SSD) has been defined as a sub-compartment within the synaptic complex in which the density of a given protein is higher than in the surrounding area (Yang \& Specht, 2019). Here, we have shown that several components of mixed inhibitory synapses in spinal cord neurons form SSDs, including RIM1/2, gephyrin, GlyRs and GABA $A_{A}$.

To exclude that an inherent stochasticity of dSTORM imaging could be behind the observation of SSDs, we have considered in our experiments the number of detections per secondary antibody (50.4 \pm 3.3 for IgG-Alexa 647 , and $47.6 \pm 3.3$ for IgG-Cy3B, mean \pm SEM, see (Yang \& Specht, 2020)) and extrapolated this value to estimate the number of sampled synaptic receptors. Accordingly, the number of detected $\mathrm{GABA}_{A} \mathrm{Rs}$ (IgG-Alexa 647) per synapse in the control condition was estimated to be roughly 20 . The number of detected GlyRs (IgG-Cy3B) per synapse was $\sim 29$. The average number of receptors per SSD was 5 for $\mathrm{GABA}_{A} \mathrm{Rs}$ and 8 for GlyRs in the control. Increased network activity by 4-AP treatment reduced the number of detected $\mathrm{GABA}_{A} \mathrm{Rs}$ to 8 per synapse and to 3 per SSD, without affecting the number of GlyRs (23 per synapse, 8 per SSD). Although these values are lower than the absolute copy numbers at synapses (Liu et al., 2020, Nusser et al., 1997, Patrizio et al., 2017), the efficiency of the immuno-labeling nonetheless allows for an accurate structural description.

We found that pre-synaptic RIM1/2 SSDs and post-synaptic gephyrin SSDs are aligned in transsynaptic nanocolumns at mixed inhibitory synapses. This arrangement is similar to that observed at excitatory synapses, consisting of RIM1/2 and the post-synaptic scaffold protein PSD-95 (Tang et al., 2016). Moreover, GlyR SSDs and GABA $A_{A} R$ SSDs were also aligned with the SSDs of RIM1/2. This 
suggests that GlyRs and $G A B A_{A} R s$ are integrated into inhibitory the trans-synaptic nanocolumns. Modeling predicts that the positioning of receptors in front of vesicle release sites can increase the transmission efficacy at excitatory synapses (Haas et al., 2018, MacGillavry et al., 2013). Similarly, the alignment of GlyR and GABA ${ }_{A} R$ SSDs with RIM1/2 may ensure an effective inhibitory neurotransmission, and provide a means of regulation by slightly shifting receptors from their positions.

At mixed inhibitory synapses, the overlap between GlyR SSDs and GABA $A_{A} R$ SSDs was surprisingly low, suggesting that the receptor domains are distinct, overlapping only at a subset of synapses in spinal cord neurons. This pattern is reminiscent of the differential distribution of AMPARs and GluN2A and GluN2B-containing NMDARs at excitatory synapses, where the nanoscale organization of the receptors may regulate access to released glutamate (Kellermayer et al., 2018, MacGillavry et al., 2013). In the case of inhibitory synapses, the distribution of GlyRs and $G A B A_{A} R s$ SSDs could have a similar impact on their activation, depending on which SSDs are closest to the vesicle release sites, as suggested by the detection of purely glycinergic and GABAergic mIPSCs in young spinal cord neurons (Keller et al., 2001). However, the situation could be more complicated at mixed inhibitory synapses, since GlyRs and $G A B A_{A} R s$ are activated by different neurotransmitters, both of which are released from the same pre-synaptic vesicles (Aubrey \& Supplisson, 2018, Jonas et al., 1998). The relative vesicle load and binding affinity of the neurotransmitters therefore add to the complexity related to the nanoscale organization that controls the distance of GlyRs and $G A B A_{A} R s$ to the release site.

It is generally accepted that gephyrin molecules are organized into a relatively stable lattice at inhibitory PSDs (Alvarez, 2017, Sola et al., 2004). We found that the gephyrin SSDs contain high levels of phosphorylated gephyrin molecules (pS270; Figures 2 and 7). SSDs of pS270 gephyrin have also been detected in previous studies (Crosby et al., 2019, Niwa et al., 2019, Pennacchietti et al., 2017). This raises the concept that the ratio between total and phosphorylated pS270 gephyrin is a key regulatory switch at inhibitory synapses. Given that gephyrin phosphorylation was reduced in response to 4-AP treatment without altering total gephyrin levels, it is possible that pS270 gephyrin is concentrated within sub-domains of the gephyrin scaffold at mixed inhibitory synapses. This is confirmed by the observation that the sub-synaptic pS270 distribution does not change with 4-AP (Figure 7). In other words, the loss of pS270 signal appears to occur in regions of the PSD that do not form part of the SSDs. The relative stability of gephyrin phosphorylation inside and out of SSDs may thus provide a potential mechanism for the differential regulation of GlyR and $G A B A_{A} R$ clustering at mixed inhibitory synapses. 
When network activity levels were altered, the nanoscale organization of GlyRs and $G A B A_{A} R s$ was differentially adjusted (Figures 5 and 6). While $\mathrm{GABA}_{A} \mathrm{R}$ clustering was reduced in response to higher activity after 4-AP treatment, GlyRs remained largely unchanged. GlyR levels may be sustained by the gephyrin scaffold independently of changes of its phosphorylation status due to the high binding affinity of the GlyRß subunit for gephyrin. In contrast, de-phosphorylation of gephyrin outside of SSDs could be responsible for the homeostatic reduction of $G A B A_{A} R s$ under 4-AP. This is in agreement with the reduced $G A B A_{A} R$ clustering downstream of gephyrin de-phosphorylation induced by cAMP signaling (Niwa et al., 2019). Taken together, our results demonstrate that GlyRs and $G A B A_{A} R s$ are differentially regulated by gephyrin through different signaling pathways. The fact that GlyRs and $G A B A_{A} R s$ occupy distinct sub-synaptic domains and are regulated by different signaling pathways provides a new angle for the understanding of co-transmission at mixed inhibitory synapses in spinal cord neurons.

\section{Acknowledgements}

We thank Marianne Renner for providing the pickpointsSR program, Philippe Rostaing and Oliver Gemin for preparing cryosections, Felipe Delestro and Auguste Genovesio for technical support in data analysis. Funding was provided by the European Research Council (ERC, Plastinhib), Agence Nationale de la Recherche (ANR, Synaptune and Syntrack), Labex (Memolife) and France-Biolmaging (FBI). XY was supported by the China Scholarship Council.

\section{The authors declare that they have no conflict of interest.}

\section{Methods}

\section{Primary spinal cord neuron culture}

All procedures using animals follow the regulations of the French Ministry of Agriculture and the Direction départementale des services vétérinaires de Paris (Ecole Normale Supérieure, animalerie des rongeurs, license B 75-05-20). Primary spinal cord neurons were prepared from embryonic Sprague Dawley rats on embryonic day E14 as described (Specht et al., 2013), with some modifications. Dissociated neurons were plated on $18 \mathrm{~mm}$ glass coverslips (thickness $0.16 \mathrm{~mm}$, No. $1.5, \mathrm{VWR} \# 6310153)$ that were pre-washed with ethanol and coated with $70 \mu \mathrm{g} / \mathrm{ml}$ poly-DL- 
ornithine. Cells were seeded at a density of $6 \times 10^{4}$ cells $/ \mathrm{cm}^{2}$ in Neurobasal medium (ThermoFisher), supplemented with B-27, $2 \mathrm{mM}$ glutamine, and antibiotics $(5 \mathrm{U} / \mathrm{ml}$ penicillin and $5 \mathrm{\mu g} / \mathrm{ml}$ streptomycin). Neurons were cultured at $37^{\circ} \mathrm{C}$ with $5 \% \mathrm{CO}_{2}$, and the medium was replenished twice a week by replacing half of the volume with BrainPhys neuronal medium (Stemcell Technologies) supplemented with SM1 and antibiotics. Cells were used for experiments between DIV14 and DIV21.

\section{Calcium imaging}

Spinal cord neurons were loaded with 0.5 MM Fluo-4 AM (F14201, Life Technologies) diluted in culture medium and incubated for $10 \mathrm{~min}$. Cells were then imaged within pre-warmed imaging medium, containing $130 \mathrm{mM} \mathrm{NaCl}, 5 \mathrm{mM} \mathrm{KCl}, 2 \mathrm{mM} \mathrm{CaCl}, 1 \mathrm{mM} \mathrm{MgCl}, 30 \mathrm{mM}$ glucose, and $10 \mathrm{mM}$ HEPES, pH 7.4. Time lapse recordings were acquired for $3 \mathrm{~min}$ at $10 \mathrm{~Hz}$ before and after application of TTX $(1 \mu \mathrm{M})$ or 4-AP $(50 \mu \mathrm{M})$, respectively. Background corrected calcium signals were measured in cell bodies using Fiji (Schindelin et al., 2012). The frequency and peak amplitude was detected using a custom written program (courtesy from Anastasia Ludwig) in Matlab (MathWorks).

\section{Pharmacological treatment and immunocytochemistry}

Cultured neurons were treated for one hour with tetrodotoxin citrate (TTX, Cat. No. 1069, Tocris) or 4-aminopyridine (4-AP, Cat. No. 0940, Tocris), diluted in culture medium at a final concentration of 1 $\mu \mathrm{M}$ and $50 \mu \mathrm{M}$, respectively. The cultures were then fixed with $100 \%$ methanol at $-20^{\circ} \mathrm{C}$ for 10 minutes. Unspecific binding sites were blocked with 3\% BSA in PBS (blocking buffer) for at least 30 minutes. Cells were sequentially incubated for 1 hour at room temperature with primary antibodies and with secondary antibodies diluted in blocking solution. Each step was followed by several washes with PBS.

The following primary antibodies were used: mouse monoclonal mAb7a (m7a, Synaptic Systems, \#147011), rat monoclonal mAb7a (rb7a, Synaptic Systems, \#147208), and rabbit polyclonal (rbGPHN, Synaptic Systems, \#147002) antibodies against gephyrin; mouse monoclonal antibody against GABA $_{A} R$ R3 (UC Davis/NIH NeuroMab, \#75-149, RRID: AB_2109585), rabbit polyclonal antibody against RIM1/2 (Synaptic Systems, \#140203), guinea pig polyclonal antibody against RIM1/2 (Synaptic Systems, \#140205), and custom-made rabbit polyclonal antibody against GlyR $\alpha 1$ (Triller lab, \#2353). The primary antibodies were used at a dilution of 1:500, following the manufacturer's instructions. The following commercial secondary antibodies were used: Alexa Fluor 647-conjugated donkey anti-guinea pig IgG (Jackson ImmunoResearch, Cat. No. 706-605-148), donkey anti-rabbit (Jackson, Cat. No. 711-605-152), donkey anti-mouse (Jackson, Cat. No. 715-605-151) and goat antirat IgG (Invitrogen, Cat. No. A21247); Cy3-conjugated goat anti-rabbit (Jackson, Cat. No. 111-165- 
144) and goat anti-mouse IgG (Jackson, Cat. No. 115-165-166); Alexa 488-conjugated goat antimouse IgG (Jackson, Cat. No. 115-545-166). Secondary antibodies were diluted at 1:500 or 1:1000, following the manufacturers' recommendations. Secondary antibodies that had been conjugated with Cy3B in our lab were diluted at 1:50 or 1:100 to compensate for the dilution during the purification process.

\section{Conjugation of secondary antibodies with Cy3B dye}

Unconjugated secondary antibodies, donkey anti-mouse IgG (Jackson ImmunoResearch, \#715-005151), donkey anti-rabbit IgG (Jackson ImmunoResearch, \#711-005-152), donkey anti-guinea pig IgG (Jackson ImmunoResearch, \#706-005-148) were coupled with Cy3B mono-reactive NHS ester (PA63101, GE Healthcare) according to the supplier's protocol. Antibodies were then purified using size exclusion columns (Illustra NAP-5 columns, \#17085302, GE Healthcare). The absorption of IgG at $280 \mathrm{~nm}$ and Су3В dye at $559 \mathrm{~nm}$ of the reaction products was measured by spectrophotometry (NanoDrop ND-1000 Spectrophotometer), from which the number of dyes per IgG was calculated. The estimated dye/IgG ratio was generally between 3 and 5 .

\section{Cryosection preparation and immunohistochemistry}

Adult male mice (C57BL/6J, 10 weeks old) were deeply anesthetized with pentobarbital, and intracardially perfused with 4\% PFA and $0.1 \%$ glutaraldehyde in PBS. The cervical to thoracic spinal cord segments were dissected and post-fixed in $4 \%$ PFA at $4^{\circ} \mathrm{C}$ overnight. The segments were then cut into $1 \mathrm{~mm}^{3}$ cubes and incubated in $2.3 \mathrm{M}$ sucrose at $4^{\circ} \mathrm{C}$ for 2-3 days. The tissue was then cut into sections of $2 \mu \mathrm{m}$ thickness with an ultra-microtome (Leica EM UC6). Sections were placed on glass coverslips for immunohistochemistry.

Sucrose impregnated cryosections were first subjected to a heat-induced antigen retrieval (HIAR) protocol (Rousseau et al., 2012). Briefly, sections were immersed in a citrate-based antigen retrieval buffer and placed in a de-cloaking chamber (Biocare Medical) for $20 \mathrm{~min}$ at $110^{\circ} \mathrm{C}$. They were then treated with $15 \%$ methanol and $0.3 \% \mathrm{H}_{2} \mathrm{O}_{2}$ in PBS for $15 \mathrm{~min}$, followed by $1 \%$ sodium borohydride in PBS for 45 min. Sections were thoroughly rinsed with PBS after each step. Unspecific binding sites were blocked with 3\% BSA in PBS at room temperature for at least two hours. Primary antibodies, including rabbit polyclonal antibody against RIM1/2 and mouse monoclonal mAb7a, were diluted in blocking buffer and applied overnight at $4^{\circ} \mathrm{C}$. Sections were then incubated with Alexa 647conjugated donkey anti-rabbit IgG (Jackson ImmunoResearch, Cat. No. 711-605-152, dilution 1:500) and lab-made Cy3B-conjugated donkey anti-mouse secondary antibodies (dilution 1:100) for two hours at room temperature. 


\section{Conventional fluorescence microscopy and image analysis}

After immuno-labeling, coverslips were mounted in an open chamber in PBS. Images were acquired on an inverted Nikon Eclipse Ti microscope, equipped with a 100× oil immersion objective (HP APO TIRF 100× oil, NA 1.49, Nikon). A mercury lamp (Intensilight C-HGFIE, Nikon) was used for illumination, with specific band pass filters for the far-red (excitation FF01-650/13, emission FF02684/24, Semrock), red (ex. FF01-560/25, em. FF01-607/36) and green channels (ex. FF02-485/20, em. FF01-525/30). Ten image frames of $100 \mathrm{~ms}$ or $200 \mathrm{~ms}$ exposure (depending on the channel) were recorded for each field of view. For a given set of experiments, all imaging parameters were kept constant between all experimental conditions. The stacks of images were combined by average projection of the ten frames in Fiji. Binary masks were then produced from the gephyrin immunolabelling using the wavelet function in Icy (de Chaumont et al., 2012), to measure the intensities of all the different labeled proteins within the synaptic cluster. The integrated intensity within the binary masks was determined using a lab-made program (Hennekinne et al., 2013) written in Matlab.

\section{Sequential two-color dSTORM imaging}

The dSTORM setup is built on the same Nikon Ti microscope described above. It includes several continuous laser lines with emission wavelengths at $640 \mathrm{~nm}, 561 \mathrm{~nm}$ and $405 \mathrm{~nm}$ (Coherent) with nominal maximum power of $1 \mathrm{~W}, 1 \mathrm{~W}$, and $120 \mathrm{~mW}$, respectively. The setup is equipped with a total internal reflection fluorescence (TIRF) arm to set the angle of the illumination laser, an acousto-optic tunable filter (AOTF) to control the intensity of the lasers, a perfect focusing system (PFS) to maintain the focal plane during imaging, and an electron multiplying charge-coupled device (EMCCD) camera (Andor iXon Ultra) for image acquisition. All elements of the microscope are controlled by NIS-Elements software (Nikon). The dSTORM recordings were taken at a magnification of 100x, resulting in image pixel size of $160 \mathrm{~nm}$.

On the day of imaging, immuno-labeled neurons on glass coverslips were incubated with $100 \mathrm{~nm}$ beads (Tetraspeck, ThermoFisher, Cat. No. T7279), and mounted on glass slides with a cavity (diameter $15-18 \mathrm{~mm}$, depth 0.6-0.8 $\mathrm{mm}$ ) containing freshly prepared Gloxy imaging buffer (0.5 $\mathrm{mg} / \mathrm{ml}$ glucose oxidase, $40 \mu \mathrm{g} / \mathrm{ml}$ catalase, $0.5 \mathrm{M}$ D-glucose, $50 \mathrm{mM} \beta$-mercaptoethylamine (MEA) in PBS, pH 7.4, degassed with nitrogen). The coverslips were sealed with silicone rubber (Picodent twinsil speed 22) and the slide was mounted on the microscope. Prior to dSTORM recordings, conventional epifluorescence images of each field of view were taken with the mercury lamp (Figure S2A1-D1). These epifluorescence images were used as reference during the dSTORM data 
processing. Sequential two-color ASTORM imaging was then carried out first in the far-red channel (Alexa 647), followed by the red channel (Сy3B). No UV light was applied during the recording of Alexa 647, however, the blinking of Cy3B was supported by low-intensity $405 \mathrm{~nm}$ laser illumination. Highly inclined illumination was used to reduce the background fluorescence. Recordings of 20000 or 30000 frames with an exposure time of $50 \mathrm{~ms}$ per frame were acquired. The frame numbers were kept constant for each set of experiments. For single-color dSTORM imaging, only the signals of Alexa 647 in the far-red channel were collected.

\section{dSTORM data processing}

Processing steps of the imaging data included single particle detection, lateral drift correction, twochannel registration and image reconstruction (Yang \& Specht, 2020). Single particle detection was realized by applying Gaussian fitting to the PSF of each fluorophore signal, using an adapted version of the multiple-target tracing (MTT) program (Serge et al., 2008) in Matlab. We obtained a localization precision of $6 \pm 3 \mathrm{~nm}$ for Alexa 647 signals, and $7 \pm 2 \mathrm{~nm}$ for Cy3B, by the approximation of $\Delta / \mathrm{VN}$, where $\Delta$ is the full width at half-maximum (FWHM) of the point spread function (PSF) and $N$ is the number of collected photons. Lateral drift was corrected in the Matlab program PALMvis (Lelek et al., 2012) using beads as fiducial markers. At this stage, we obtained the coordinates-based localization data in both channels (Figure S2A2-D2). The drift-corrected coordinates were then rendered into super-resolution dSTORM images (pixel size $10 \mathrm{~nm}$ ) by representing each localization as a Gaussian function with a standard deviation $\sigma=15 \mathrm{~nm}$ (Figure S2A3-D3). Image rendering was done either with PALMvis to obtain a well-defined structural representation or with LAMA software (Malkusch \& Heilemann, 2016) if a consistent representation of detection numbers was required.

\section{SSD segmentation and feature extraction}

To define the regions of interest (mixed inhibitory synapses), individual synaptic clusters were cropped from the rendered images using binary masks produced from the epifluorescence images. SSDs were segmented from these individual synaptic clusters using the $\mathrm{H}$-watershed plugin developed by Benoit Lombardot (http://imagej.net/Interactive_Watershed) in Fiji. The SSD counts per synaptic cluster and SSD area were extracted using extended particle analyzer (Brocher, 2014) in Fiji.

\section{Quantification of SSD distances and alignment}

Rendered dSTORM images of the two proteins of interest were composed and transformed to RGB format in Fiji. SSD distances and alignment were only measured in synapses seen in cross-section. We manually selected these side views following the following criteria: clusters showing obvious 
elongated shapes in both channels, or synapses with elongated shapes in only one of the two channels and with clear signals in the other channel. After SSD segmentation, the paired SSDs of two different proteins were identified. In the RGB images, a line was drawn through the intensity peaks of the paired SSDs and the distance between the peaks was taken as SSD distance. When there was more than one pair of SSDs per synapse, the average of all measured distances was taken as SSD distance for that synapse. Paired SSDs of pre-synaptic and post-synaptic proteins were counted manually. For each protein, the degree of alignment was calculated as the number of paired SSDs divided by the total number of SSDs for that protein, values expressed on a scale from 0 to 1 (fraction of aligned SSDs).

\section{Counting dSTORM detections per synapse and per SSD}

To obtain the binary masks of synaptic area, the rendered images from the two dSTORM channels generated with LAMA software were combined and binarized without thresholding. For one-color dSTORM, the rendered images were binarized directly to obtain synaptic masks. Binary SSD images were produced with the $\mathrm{H}$-watershed plugin in Fiji. The detection coordinates were then overlaid with the binary images and detections within the synaptic masks or SSDs were counted using the pickpointsSR program (courtesy from Marianne Renner, IFM, Paris) written in Matlab (Figure S2A4D4).

\section{Statistics}

Statistical analysis was generally done using the non-parametric Mann-Whitney U-test (MW), Kolmogorov-Smirnov test (KS), and Friedman test followed by a Dunn's post-hoc multiple comparison test (in Figure S3C). Where the data passed a D'Agostino \& Pearson normality test, the analysis was done with a Student's t-test or one-way ANOVA followed by Tukey's post-hoc test. Data are represented in the form of cumulative probability distributions, or as bar graphs showing the mean \pm SEM (or the median and the quartiles of the distribution in Figure S3) unless otherwise stated. 


\section{References}

Alvarez FJ (2017) Gephyrin and the regulation of synaptic strength and dynamics at glycinergic inhibitory synapses. Brain Res Bull 129: 50-65

Aubrey KR, Supplisson S (2018) Heterogeneous signaling at GABA and glycine co-releasing terminals. Front Synaptic Neurosci 10: 40

Brocher J (2014) Qualitative and quantitative evaluation of two new histogram limiting binarization algorithms. International Journal of Image Processing (IJIP) 8: 30-48

Cantaut-Belarif $Y$, Antri M, Pizzarelli R, Colasse S, Vaccari I, Soares S, Renner M, Dallel R, Triller A, Bessis A (2017) Microglia control the glycinergic but not the GABAergic synapses via prostaglandin E2 in the spinal cord. J Cell Biol 216: 2979-2989

Chery N, de Koninck Y (1999) Junctional versus extrajunctional glycine and GABA(A) receptormediated IPSCs in identified lamina I neurons of the adult rat spinal cord. J Neurosci 19: 7342-55

Crosby KC, Gookin SE, Garcia JD, Hahm KM, Dell'Acqua ML, Smith KR (2019) Nanoscale subsynaptic domains underlie the organization of the inhibitory synapse. Cell Rep 26: 3284-3297 e3

de Chaumont F, Dallongeville S, Chenouard N, Herve N, Pop S, Provoost T, Meas-Yedid V, Pankajakshan P, Lecomte T, Le Montagner Y, Lagache T, Dufour A, Olivo-Marin JC (2012) Icy: an open bioimage informatics platform for extended reproducible research. Nat Methods 9: 690-6

Dempsey GT, Vaughan JC, Chen KH, Bates M, Zhuang X (2011) Evaluation of fluorophores for optimal performance in localization-based super-resolution imaging. Nat Methods 8: 1027-36

Dumoulin A, Levi S, Riveau B, Gasnier B, Triller A (2000) Formation of mixed glycine and GABAergic synapses in cultured spinal cord neurons. Eur J Neurosci 12: 3883-92

Ghosh H, Auguadri L, Battaglia S, Simone Thirouin Z, Zemoura K, Messner S, Acuna MA, Wildner H, Yevenes GE, Dieter A, Kawasaki H, M OH, Zeilhofer HU, Fritschy JM, Tyagarajan SK (2016) Several posttranslational modifications act in concert to regulate gephyrin scaffolding and GABAergic transmission. Nat Commun 7: 13365

Haas KT, Compans B, Letellier M, Bartol TM, Grillo-Bosch D, Sejnowski TJ, Sainlos M, Choquet D, Thoumine O, Hosy E (2018) Pre-post synaptic alignment through neuroligin-1 tunes synaptic transmission efficiency. Elife 7

Hennekinne L, Colasse S, Triller A, Renner M (2013) Differential control of thrombospondin over synaptic glycine and AMPA receptors in spinal cord neurons. J Neurosci 33: 11432-9

Jonas P, Bischofberger J, Sandkuhler J (1998) Corelease of two fast neurotransmitters at a central synapse. Science 281: 419-24

Kalbouneh H, Schlicksupp A, Kirsch J, Kuhse J (2014) Cyclin-dependent kinase 5 is involved in the phosphorylation of gephyrin and clustering of GABAA receptors at inhibitory synapses of hippocampal neurons. PLoS One 9: e104256

Keller AF, Coull JA, Chery N, Poisbeau P, De Koninck Y (2001) Region-specific developmental specialization of GABA-glycine cosynapses in laminas I-II of the rat spinal dorsal horn. J Neurosci 21: 7871-80

Kellermayer B, Ferreira JS, Dupuis J, Levet F, Grillo-Bosch D, Bard L, Linarès-Loyez J, Bouchet D, Choquet D, Rusakov DA, Bon P, Sibarita JB, Cognet L, Sainlos M, Carvalho AL, Groc L (2018) Differential Nanoscale Topography and Functional Role of GluN2-NMDA Receptor Subtypes at Glutamatergic Synapses. Neuron 100: 106-119.e7

Kneussel M, Betz $\mathrm{H}$ (2000) Clustering of inhibitory neurotransmitter receptors at developing postsynaptic sites: the membrane activation model. Trends Neurosci 23: 429-35 
Kuhse J, Kalbouneh H, Schlicksupp A, Mukusch S, Nawrotzki R, Kirsch J (2012) Phosphorylation of gephyrin in hippocampal neurons by cyclin-dependent kinase CDK5 at Ser-270 is dependent on collybistin. J Biol Chem 287: 30952-66

Legendre P (2001) The glycinergic inhibitory synapse. Cell Mol Life Sci 58: 760-93

Lelek M, Di Nunzio F, Henriques R, Charneau P, Arhel N, Zimmer C (2012) Superresolution imaging of HIV in infected cells with FIAsH-PALM. Proc Natl Acad Sci U S A 109: 8564-9

Levi S, Schweizer C, Bannai H, Pascual O, Charrier C, Triller A (2008) Homeostatic regulation of synaptic GlyR numbers driven by lateral diffusion. Neuron 59: 261-73

Liu YT, Tao CL, Zhang X, Xia W, Shi DQ, Qi L, Xu C, Sun R, Li XW, Lau PM, Zhou ZH, Bi GQ (2020) Mesophasic organization of GABA(A) receptors in hippocampal inhibitory synapses. Nat Neurosci

MacGillavry Harold D, Song Y, Raghavachari S, Blanpied Thomas A (2013) Nanoscale Scaffolding Domains within the Postsynaptic Density Concentrate Synaptic AMPA Receptors. Neuron 78: 615622

Maidorn M, Rizzoli SO, Opazo F (2016) Tools and limitations to study the molecular composition of synapses by fluorescence microscopy. Biochem J 473: 3385-3399

Malkusch S, Heilemann M (2016) Extracting quantitative information from single-molecule superresolution imaging data with LAMA - LocAlization Microscopy Analyzer. Sci Rep 6: 34486

Nair D, Hosy E, Petersen JD, Constals A, Giannone G, Choquet D, Sibarita JB (2013) Super-resolution imaging reveals that AMPA receptors inside synapses are dynamically organized in nanodomains regulated by PSD95. J Neurosci 33: 13204-24

Niwa F, Patrizio A, Triller A, Specht CG (2019) cAMP-EPAC-Dependent Regulation of Gephyrin Phosphorylation and GABAAR Trapping at Inhibitory Synapses. iScience 22: 453-465

Nusser Z, Cull-Candy S, Farrant M (1997) Differences in synaptic GABA(A) receptor number underlie variation in GABA mini amplitude. Neuron 19: 697-709

O'Brien JA, Berger AJ (2001) The nonuniform distribution of the GABA(A) receptor alpha 1 subunit influences inhibitory synaptic transmission to motoneurons within a motor nucleus. J Neurosci 21: 8482-94

Patriarchi T, Buonarati OR, Hell JW (2018) Postsynaptic localization and regulation of AMPA receptors and Cav1.2 by $\beta 2$ adrenergic receptor/PKA and $\mathrm{Ca}(2+) / \mathrm{CaMKII}$ signaling. Embo j 37

Patrizio A, Renner M, Pizzarelli R, Triller A, Specht CG (2017) Alpha subunit-dependent glycine receptor clustering and regulation of synaptic receptor numbers. Sci Rep 7: 10899

Pennacchietti F, Vascon S, Nieus T, Rosillo C, Das S, Tyagarajan SK, Diaspro A, Del Bue A, Petrini EM, Barberis A, Cella Zanacchi F (2017) Nanoscale molecular reorganization of the inhibitory postsynaptic density is a determinant of GABAergic synaptic potentiation. J Neurosci 37: 17471756

Rousseau CV, Dugué GP, Dumoulin A, Mugnaini E, Dieudonné S, Diana MA (2012) Mixed inhibitory synaptic balance correlates with glutamatergic synaptic phenotype in cerebellar unipolar brush cells. J Neurosci 32: 4632-44

Saiyed T, Paarmann I, Schmitt B, Haeger S, Sola M, Schmalzing G, Weissenhorn W, Betz H (2007) Molecular basis of gephyrin clustering at inhibitory synapses: role of $\mathrm{G}$ - and $\mathrm{E}$-domain interactions. J Biol Chem 282: 5625-32

Schindelin J, Arganda-Carreras I, Frise E, Kaynig V, Longair M, Pietzsch T, Preibisch S, Rueden C, Saalfeld S, Schmid B, Tinevez JY, White DJ, Hartenstein V, Eliceiri K, Tomancak P, Cardona A (2012) Fiji: an open-source platform for biological-image analysis. Nat Methods 9: 676-82

Schrader N, Kim EY, Winking J, Paulukat J, Schindelin H, Schwarz G (2004) Biochemical characterization of the high affinity binding between the glycine receptor and gephyrin. J Biol Chem 279: 18733-41 
Serge A, Bertaux N, Rigneault $H$, Marguet $D$ (2008) Dynamic multiple-target tracing to probe spatiotemporal cartography of cell membranes. Nat Methods 5: 687-694

Sola M, Bavro VN, Timmins J, Franz T, Ricard-Blum S, Schoehn G, Ruigrok RW, Paarmann I, Saiyed T, O'Sullivan GA, Schmitt B, Betz H, Weissenhorn W (2004) Structural basis of dynamic glycine receptor clustering by gephyrin. Embo J 23: 2510-9

Specht CG (2020) Fractional occupancy of synaptic binding sites and the molecular plasticity of inhibitory synapses. Neuropharmacology 169: 107493

Specht CG, Izeddin I, Rodriguez PC, El Beheiry M, Rostaing P, Darzacq X, Dahan M, Triller A (2013) Quantitative nanoscopy of inhibitory synapses: counting gephyrin molecules and receptor binding sites. Neuron 79: 308-21

Tang AH, Chen H, Li TP, Metzbower SR, MacGillavry HD, Blanpied TA (2016) A trans-synaptic nanocolumn aligns neurotransmitter release to receptors. Nature 536: 210-4

Todd AJ, Watt C, Spike RC, Sieghart W (1996) Colocalization of GABA, glycine, and their receptors at synapses in the rat spinal cord. J Neurosci 16: 974-82

Tretter V, Mukherjee J, Maric HM, Schindelin H, Sieghart W, Moss SJ (2012) Gephyrin, the enigmatic organizer at GABAergic synapses. Front Cell Neurosci 6: 23

Triller A, Cluzeaud F, Korn H (1987) gamma-Aminobutyric acid-containing terminals can be apposed to glycine receptors at central synapses. J Cell Biol 104: 947-56

Tyagarajan SK, Ghosh H, Yevenes GE, Imanishi SY, Zeilhofer HU, Gerrits B, Fritschy JM (2013) Extracellular signal-regulated kinase and glycogen synthase kinase 3 beta regulate gephyrin postsynaptic aggregation and GABAergic synaptic function in a calpain-dependent mechanism. J Biol Chem 288: 9634-47

Tyagarajan SK, Ghosh H, Yevenes GE, Nikonenko I, Ebeling C, Schwerdel C, Sidler C, Zeilhofer HU, Gerrits B, Muller D, Fritschy JM (2011) Regulation of GABAergic synapse formation and plasticity by GSK3beta-dependent phosphorylation of gephyrin. Proc Natl Acad Sci U S A 108: 379-84

van de Linde S, Löschberger A, Klein T, Heidbreder M, Wolter S, Heilemann M, Sauer M (2011) Direct stochastic optical reconstruction microscopy with standard fluorescent probes. Nat Protoc 6: 9911009

Wegner W, Mott AC, Grant SGN, Steffens H, Willig KI (2018) In vivo STED microscopy visualizes PSD95 sub-structures and morphological changes over several hours in the mouse visual cortex. Sci Rep 8: 219

Yang X, Specht CG (2019) Subsynaptic domains in super-resolution microscopy: The treachery of images. Front Mol Neurosci 12: 161

Yang X, Specht CG (2020) Practical guidelines for two-color SMLM of synaptic proteins in cultured neurons. In Single molecule microscopy in neurobiology, Okada Y, Yamamoto N (eds) pp 173-202. Springer 
Figure legends

Figure 1. Gephyrin SSDs and their alignment with pre-synaptic RIM1/2 in vivo.

(A-C) dSTORM imaging of gephyrin in sucrose impregnated cryosections of adult mouse spinal cord.

(A) dSTORM detections of gephyrin (red dots) overlaid with the epifluorescence image (white with grey background). Synaptic clusters of gephyrin in dSTORM images were identified by the epifluorescence puncta. Scale bar: $1 \mu \mathrm{m}$. (B) Enlarged pointillist images of the two gephyrin clusters indicated in (A). (C) Rendered images of the two gephyrin clusters outlined with the boundaries of the epifluorescence mask. Gephyrin SSDs are indicated with red asterisks in (B) and (C). Scale bar: $200 \mathrm{~nm}$. (D) Two-color dSTORM imaging of RIM1/2 and gephyrin in cryosections. From left to right: rendered images of RIM1/2 and gephyrin clusters showing the aligned SSDs (arrows); gephyrin SSDs segmented by watershed outlined with different colors; RIM1/2 SSDs outlined with different colors; binary SSDs of gephyrin and RIM1/2. Inhibitory synapses were identified by the gephyrin clusters in the epifluorescence images. Scale bar: $200 \mathrm{~nm}$. RIM1/2 was labeled with Alexa 647, gephyrin (mAb7a) with Cy3B in these experiments.

Figure 2. Trans-synaptic alignment at inhibitory synapses in cultured spinal cord neurons.

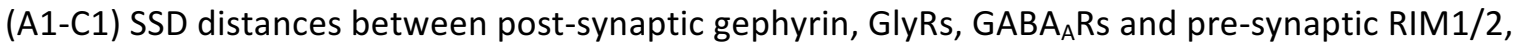
respectively. Distances were measured between the intensity peaks of paired SSDs in rendered dualcolor dSTORM images (yellow line, values given in mean \pm SEM). RIM1/2 was labeled with Alexa 647 and gephyrin (mAb7a) with Cy3B in (A1). RIM1/2 was labeled with Cy3B, while GlyRs or GABAARs were labeled with Alexa 647 in (B1) and (C1). (A2-C2) Trans-synaptic alignment of SSDs of gephyrin, GlyRs, GABA $A_{A}$ s and RIM1/2. SSDs were segmented by $\mathrm{H}$-watershed. Number of synapses: $\mathrm{n}=48$ $(A 1, A 2)$ from three independent experiments (no treatment), $n=30$ (B1, B2) from two experiments with TTX treatment, $\mathrm{n}=16(\mathrm{C} 1, \mathrm{C2})$ from two experiments with TTX. Only synapses with side view profiles were included. Scale: $200 \mathrm{~nm}$.

Figure 3. Differential organization of GlyRs and $\mathrm{GABA}_{\mathrm{A}} \mathrm{Rs}$ at mixed inhibitory synapses.

(A-B) Representative rendered dSTORM images of GlyRs and $G A B A_{A}$ Rs at synapses in side view $(A)$ or en face view (B). The lower images show the corresponding segmented SSDs. GlyRs were labelled with Alexa 647 and $G_{A B A}$ Rs with Cy3B in these experiments. Scale: $200 \mathrm{~nm}$. (C) Quantification of the overlap between GlyRs and $G_{B A}$ Rs at synapses. dSTORM images of GlyRs and GABA $A_{A}$ s were binarized, and the overlapped fraction relative to the total area occupied by the receptors was 
calculated for each synapse. (D) Overlap between the SSDs of GlyRs and GABA $A_{A}$ Rs. The fraction of SSD overlap was calculated based on the binarized images for each synapse. Over half of the synapses did not show any overlap between GlyR SSDs and GABA $A_{A} R$ SSDs (grey bar). The average overlap for the remaining synapses was $9 \% \pm 1 \%$ (mean $\pm S E M ; n=395$ synapses from three independent experiments, without selection for synapse orientation).

\section{Figure 4. Reduced $\mathrm{GABA}_{\mathrm{A}} \mathrm{R}$ levels but not GlyRs at mixed inhibitory synapses by 4-AP treatment.}

(A) Triple immuno-labeling of gephyrin, GlyRs and $G A B A_{A} R s$ at mixed inhibitory synapses (arrow) imaged with conventional fluorescence microscopy. Scale: $5 \mu \mathrm{m}$. (B) Quantification of the apparent synaptic area (in pixels) of gephyrin clusters (KS test $p<0.0001$ ). (C) Lower fluorescence intensity of $\mathrm{GABA}_{A} \mathrm{Rs}$ but only a minor reduction of GlyRs at synaptic gephyrin clusters (KS test, $p<0.0001$, both panels). (D) Reduced $G A B A_{A} R / G l y R$ intensity ratio at mixed inhibitory synapses (KS test, $p<0.0001$ ). Number of synapses: $n=9416$ in TTX and $n=6949$ in 4-AP conditions from three independent experiments.

Figure 5. Differential sub-synaptic re-organization of $\mathrm{GABA}_{A} R s$ and GlyRs by 4-AP treatment.

(A-E) Characterization of the nanoscale organization of $G_{A B A} R s$ and GlyRs at mixed inhibitory synapses. (A) Synaptic receptor cluster rendered from detections of both $G A B A_{A} R s$ (labeled with Alexa 647) and GlyRs (labeled with Cy3B). (B) Detections of $\mathrm{GABA}_{A} \mathrm{Rs}$ (red dots) and GlyRs (blue dots) overlaid with binary synaptic mask (white). (C-E) Detections of $G_{A B A} R s$, but not GlyRs were strongly decreased at mixed synapses after 4-AP treatment (KS test, $p<0.0001$ in $C$ and $E, p<0.001$ in D). (FJ) Sub-synaptic characterization of $G A B A_{A} R$ and GlyR domains. (F) Watershed segmentation of subsynaptic domains (SSDs). Each SSD is shown in different colors. (G) Detections of $G A B A_{A} R s$ (red dots) and GlyRs (blue dots) overlaid with SSDs (white). (H-J) Detections of $\mathrm{GABA}_{A} \mathrm{Rs}$ but not GlyRs at the same SSDs were reduced after 4-AP treatment (KS, $p<0.0001$ in $\mathrm{H}$ and J, $\mathrm{p}<0.01$ in I). Number of synapses: $n=439$ in TTX, $n=531$ in 4-AP condition, from three independent experiments. Scale: 200 $\mathrm{nm}$.

Figure 6. Reduced number of GABA ${ }_{A} R$ SSDs after 4-AP treatment.

(A) The number of receptor SSDs, segmented from the combined receptor clusters of $G A B A_{A} R s$ and GlyRs, was decreased after 4-AP treatment (MW test, $p<0.0001$ ). (B) This was mostly due to the loss of $G A B A_{A} R$ SSDs that were segmented from the rendered images of $G A B A_{A} R$ detections only (MW, $p$ $<0.0001$ ). (C) The number of GlyR SSDs (based on GlyR detections) were only marginally reduced (MW, $p<0.01$ ). Number of synapses: $n=452$ in TTX, $n=583$ in 4-AP condition, from three independent experiments. These analyses are based on the same data set as in Figure 5. 
Figure 7. Reduction of gephyrin phosphorylation at synapses but not within SSDs by 4-AP.

(A-C) Reduced immuno-reactivity of S270 phosphorylated gephyrin but not total gephyrin levels after 4-AP treatment, revealed by conventional fluorescence microscopy (KS test, $p<0.001$ in B1 and $p<0.0001$ in $B 2, p<0.0001$ in $C)$. Total gephyrin was probed with polyclonal rabbit primary antibody ( $r$ GPHN), and pS270 phosphorylated gephyrin with monoclonal mouse primary antibody (m7a). Number of synapses: $n=4040$ in TTX and $n=3818$ in 4-AP conditions from two independent experiments. Scale: $2 \mu \mathrm{m}$. (D-F) Reduced numbers of pS270 gephyrin (m7a) detections were recorded by dSTORM for the entire synaptic area ( $D$, KS test, $p<0.01)$. The number of detections of pS270 gephyrin per SSD $(E, K S, p=0.18)$ and the number of SSDs $(F, M W, p=0.11)$ were not changed by 4-AP treatment. Gephyrin was probed with mAb7a antibody and Alexa 647 dye. Number of synapses: $n=810$ in TTX and $n=727$ in 4-AP conditions from three independent experiments. Scale bar: $100 \mathrm{~nm}$. 
Figure 1

A

Detections of gephyrin overlaid with epifluorescence image

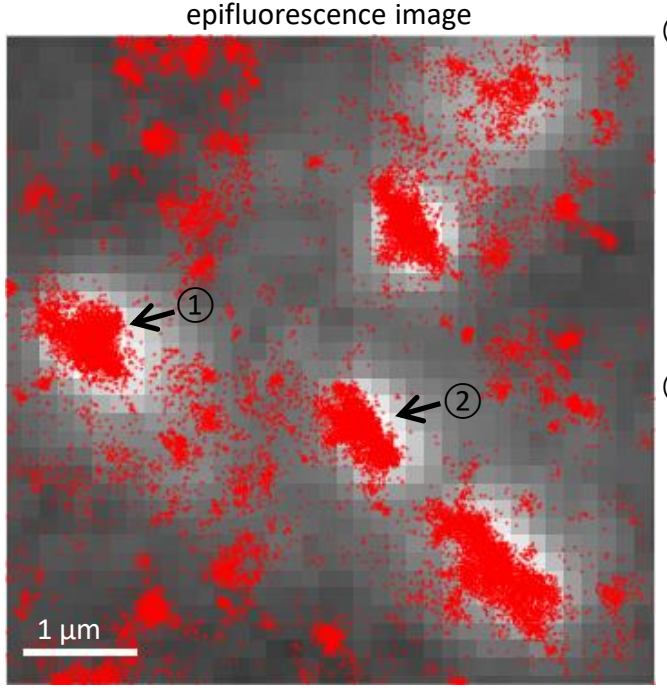

B (1)

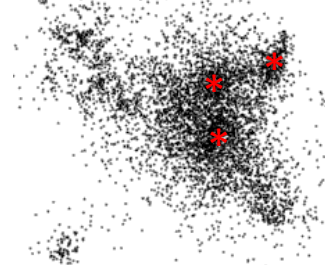

(2).

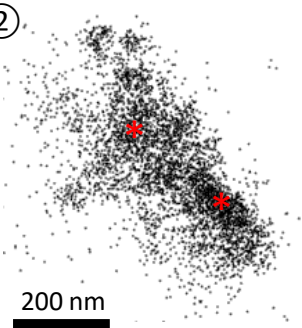

C
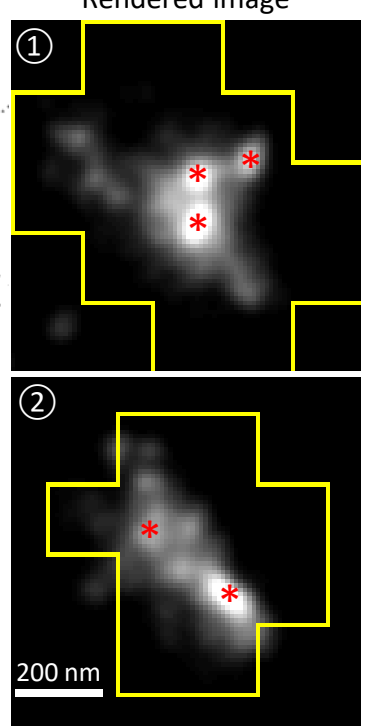

Binary SSDs of

D Gephyrin/RIM1/2

Gephyrin SSDs

RIM1/2 SSDs

Gephyrin\&RIM1/2
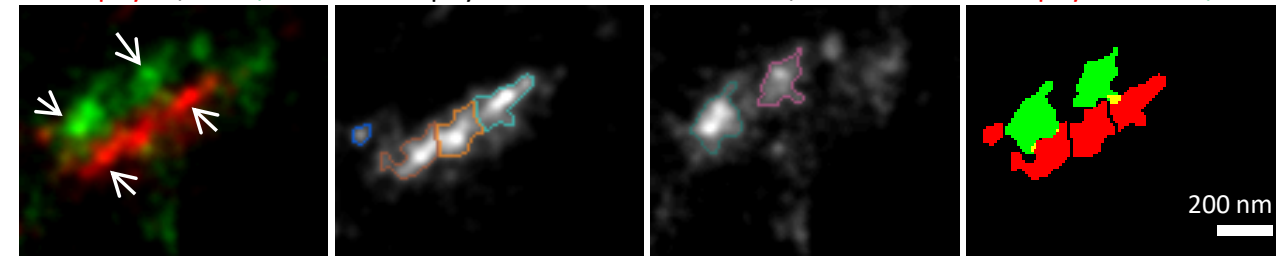
Figure 2
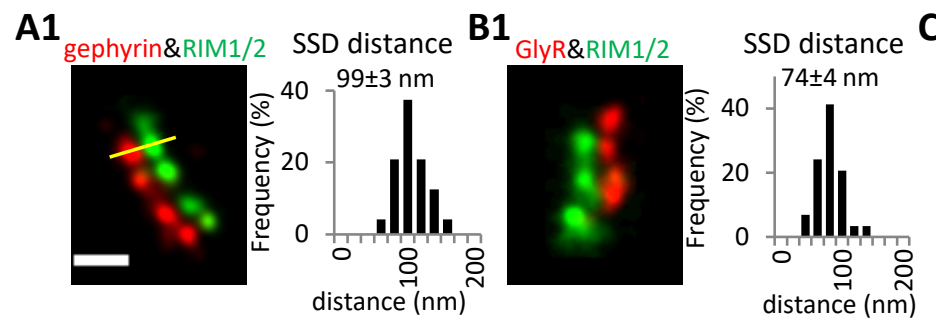

A2 H-watershed

B2 $\mathrm{H}$-watershed
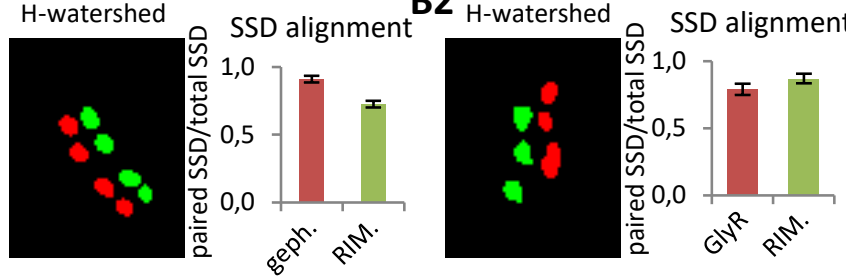

C2 $\mathrm{H}$-watershed
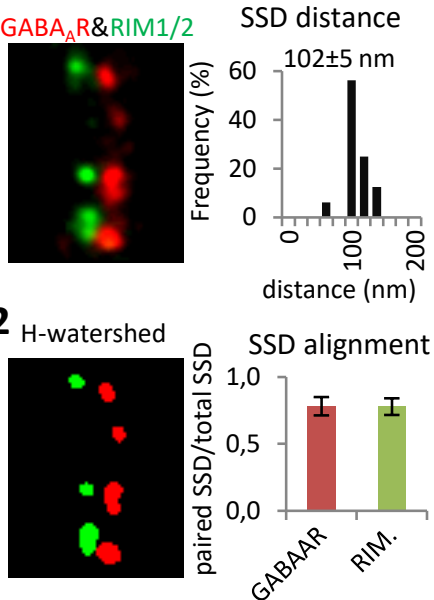
Figure 3

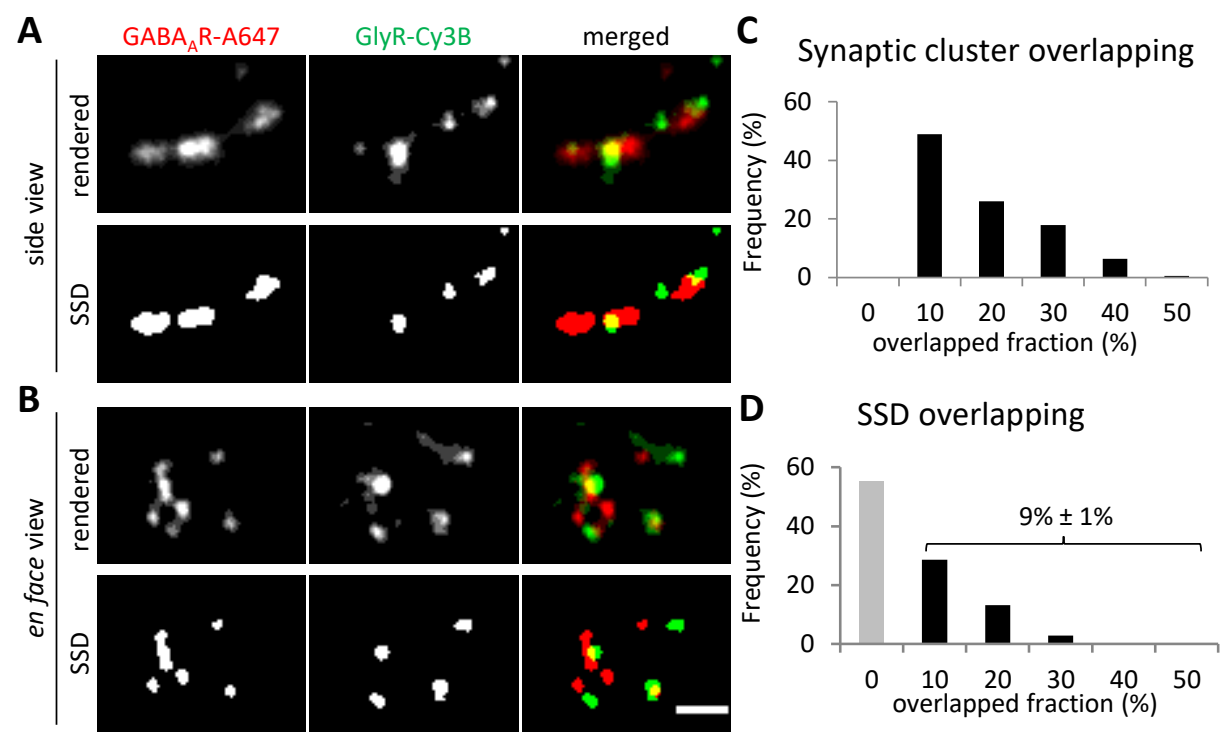


Figure 4

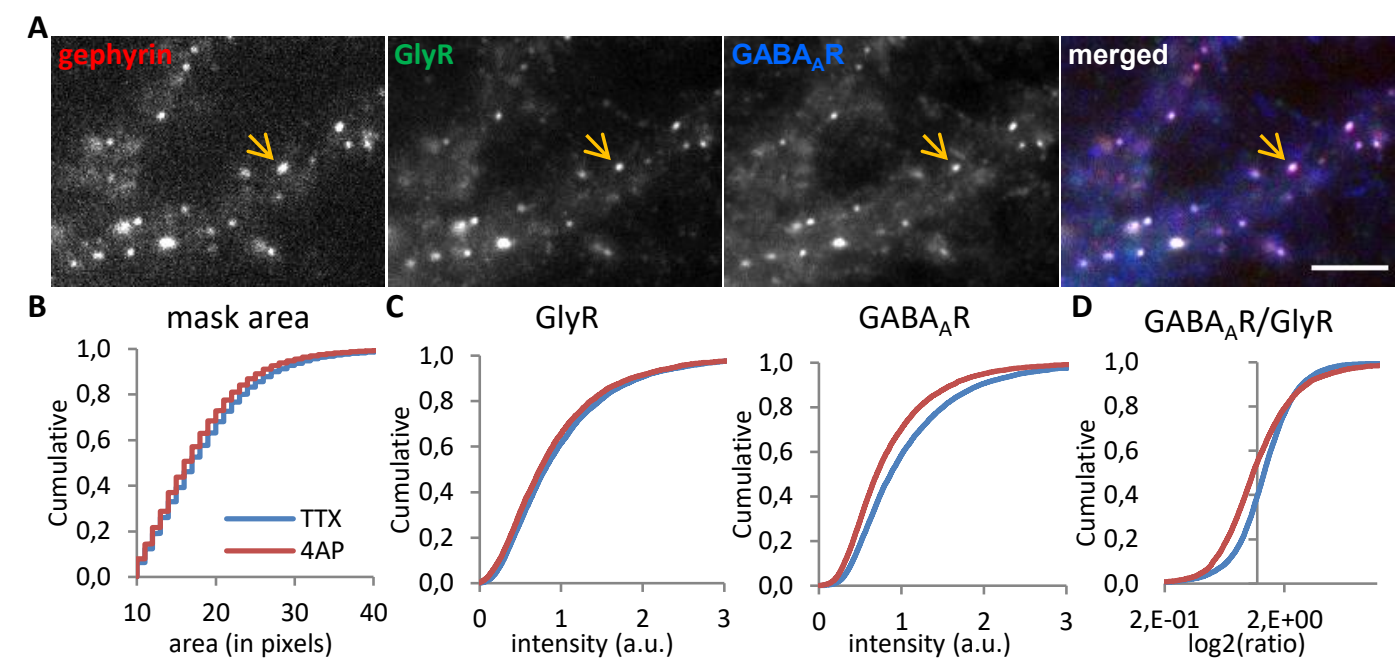


Figure 5
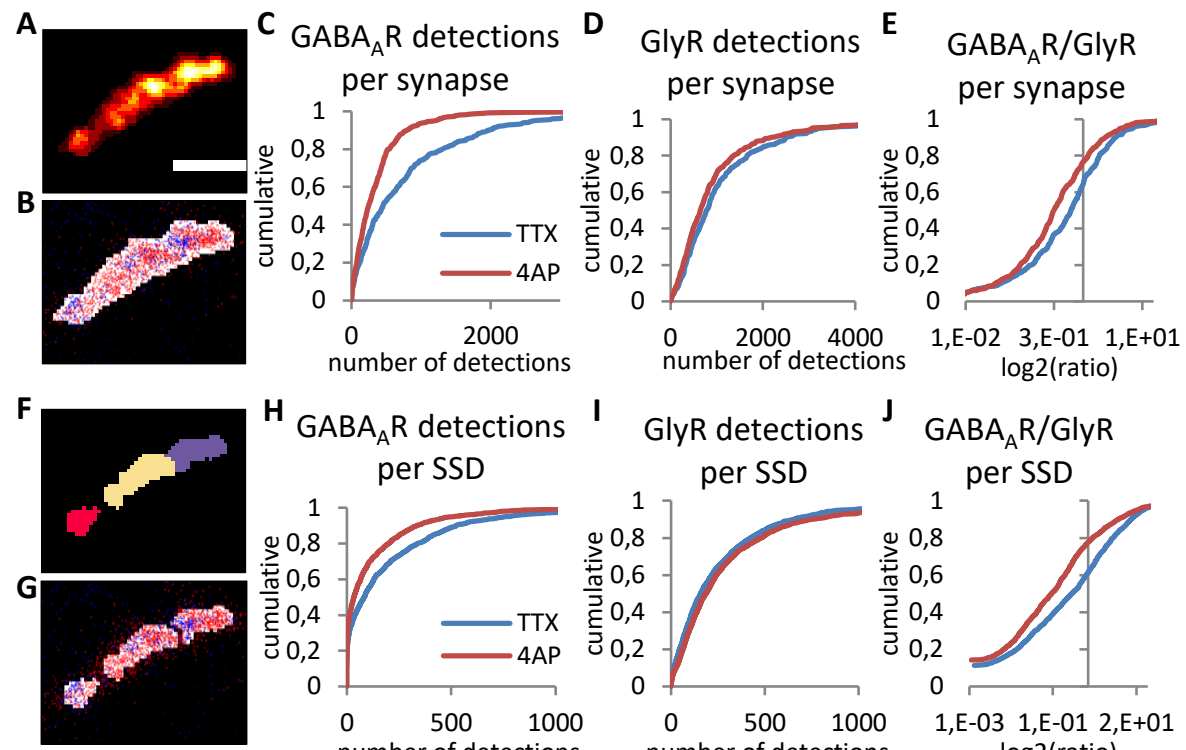

J $G A B A_{A} R / G l y R$
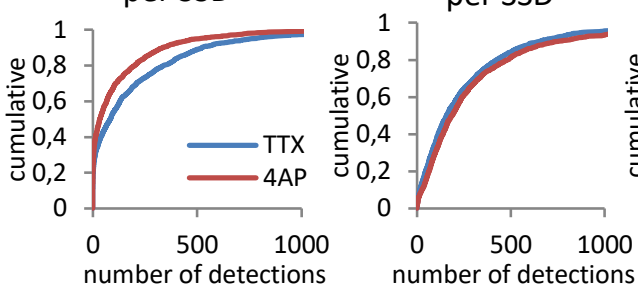

per SSD

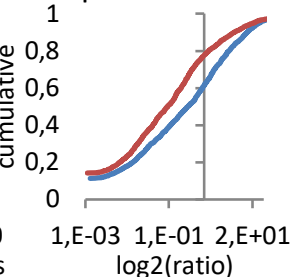


Figure 6

A Receptor SSDs B GABA ${ }_{A}$ R SSDs C GlyR SSDs

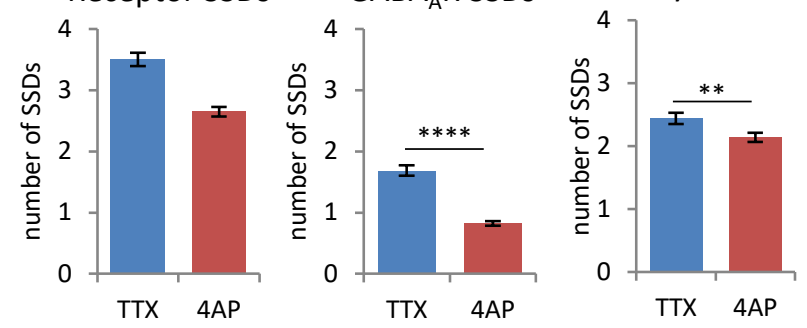


Figure 7
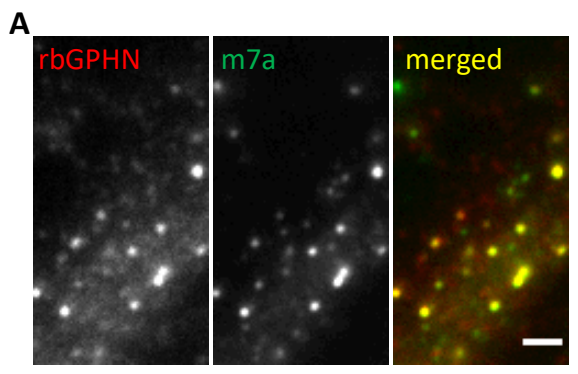

B total gephyrin gephyrin (m7a)

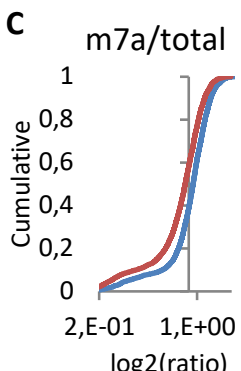

\section{D synapse}

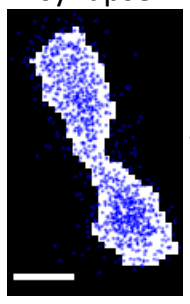

detections of m7a $\mathbf{E}$
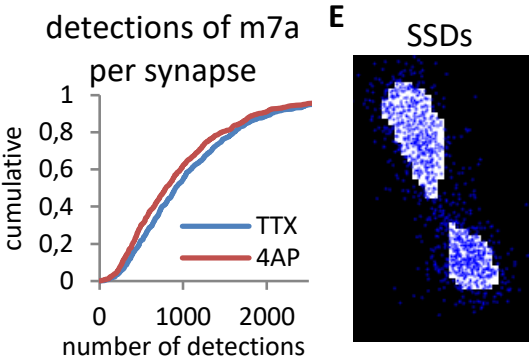

detections of $\mathrm{m} 7 \mathrm{a}$

F gephyrin SSDs
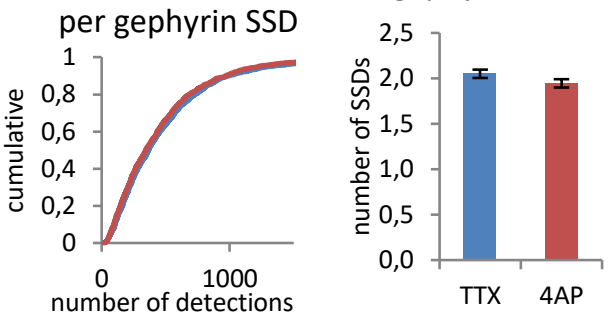